\title{
Segmentação Colaborativa de Objetos de Aprendizagem Utilizando Bookmarks em Ambientes Educacionais Ubíquos
}

\author{
Rafael D. Araújo ${ }^{1}$, Taffarel Brant-Ribeiro ${ }^{1,2}$, Hiran N. M. Ferreira ${ }^{1,2}$, \\ Fabiano A. Dorça ${ }^{1}$, Renan G. Cattelan ${ }^{1}$ \\ ${ }^{1}$ Faculdade de Computação - Universidade Federal de Uberlândia (UFU) \\ Uberlândia - Minas Gerais - Brasil \\ ${ }^{2}$ Instituto Federal do Sul de Minas Gerais (IFSULDEMINAS) \\ Passos - Minas Gerais - Brasil \\ \{rafael.araujo, fabianodor, renan\}@ufu.br, \\ \{brant.ribeiro,hiran.ferreira\}eifsuldeminas.edu.br
}

\begin{abstract}
Learning Objects (LOs) have stood out in education, supporting the teaching/learning process. However, creating LOs and their metadata is still an arduous and time-consuming task. Thus, this paper proposes a collaborative approach between students and instructors employing bookmarks to create LOs in Ubiquitous Learning Environments. A user study with 30 students using a real environment and evaluations made by three experts have been performed in order to validate our proposal. Results have showed good acceptance among students for the proposed approach and demonstrated that a set of fine-grained LOs with consistent metadata was generated, which may be reused in the future.
\end{abstract}

Resumo. Objetos de Aprendizagem (OAs) têm ganhado destaque no âmbito educacional como suporte ao processo de ensino/aprendizagem. No entanto, a criação de tais objetos e seus metadados ainda é uma tarefa árdua e que demanda tempo. Neste contexto, este trabalho apresenta uma abordagem colaborativa entre estudantes e professores utilizando bookmarks para a criação de OAs em Ambientes Educacionais Ubíquos. Um experimento com 30 usuários utilizando um ambiente real e uma avaliação com três especialistas foram realizados para validar esta proposta. Resultados demonstram que a abordagem foi bem aceita entre os estudantes e foi possivel produzir OAs com granularidade mais fina e metadados consistentes, permitindo sua reutilização no futuro.

\section{Introdução}

Objetos de Aprendizagem (OAs) são componentes instrucionais que podem assumir o papel de entidades digitais e serem utilizadas, reutilizadas e referenciadas no processo de ensino/aprendizagem em ambientes apoiados por tecnologia [IEEE 2002, Wiley 2002]. A elaboração do conteúdo desses objetos, assim como o preenchimento de seus metadados, mostra-se como um processo interdisciplinar que requer atenção a aspectos técnicos e pedagógicos, fazendo com que o mesmo se torne oneroso e demorado [Solis e Laviste 2015, Dias et al. 2009].

Neste contexto, Ambientes Educacionais Ubíquos (AEUs) [Zhao et al. 2010] surgem para proporcionar melhores experiências às pessoas envolvidas em atividades educacionais. Tais ambientes podem ser utilizados como suporte à geração de OAs para tornar 
V Congresso Brasileiro de Informática na Educação (CBIE 2016)

Anais do XXVII Simpósio Brasileiro de Informática na Educação (SBIE 2016)

mais fácil seu processo de criação [Araújo et al. 2014]. AEUs permitem que estudantes e professores utilizem ferramentas colaborativas entre si, de modo a enriquer o conteúdo disponibilizado e a experiência de aprendizagem [Brant-Ribeiro et al. 2014].

Este trabalho propõe a segmentação de OAs a partir da criação colaborativa de bookmarks em AEUs. Como validação, a abordagem proposta foi implementada em uma plataforma educacional e utilizada em um AEU real. Foram conduzidos experimentos com usuários e especialistas para avaliar a qualidade dos OAs gerados e a utilidade da funcionalidade de bookmarks colaborativos.

O restante do artigo está estruturado da seguinte maneira: a Seção 2 apresenta a fundamentação teórica acerca da utilização de bookmarks colaborativos para segmentação de OAs; na Seção 3, a abordagem proposta é apresentada juntamente com sua integração em um AEU real; a Seção 4 apresenta um experimento realizado com usuários e seus resultados; na Seção 5 são discutidos trabalhos relacionados; e, por fim, na Seção 6, são feitas as considerações finais e sugeridos trabalhos futuros.

\section{Segmentação de OAs por meio de Bookmarks Colaborativos}

Este trabalho propõe uma abordagem que utiliza os OAs criados em um AEU inicialmente apresentado em [Araújo et al. 2014]. Os OAs criados nesse ambiente representam uma sessão completa de captura (no caso, duas aulas com duração de 50 minutos cada). Metadados associados aos OAs são gerados no formato IEEE Learning Object Metadata (LOM) [IEEE 2002], porém cada OA possui uma granularidade grossa (i.e., aula completa), o que diminui a possibilidade de ser reutilizado no futuro [Braga et al. 2012].

A geração de OAs e seus metadados é uma tarefa que demanda tempo, tornando inviável a atribuição da tarefa de criação de OAs de granularidade mais fina somente aos professores. Além disso, AEUs podem ser utilizados como suporte a esse processo por meio de atividades colaborativas, visto que tais atividades são inerentes à esses ambientes. Dessa maneira, a abordagem aqui proposta utiliza ferramentas de colaboração entre professores e estudantes para a segmentação de OAs em AEUs.

Aplicações que realizam atividades de Captura e Acesso (C\&A) podem ser empregadas em AEUs com o intuito de dinamizar processos de ensino/aprendizagem. Tais aplicações são estruturadas em quatro fases essenciais: pré-produção (na qual o conteúdo é preparado), gravação "ao vivo" (quando as atividades são gravadas), pós-produção (momento em que os fluxos de mídia produzidos são armazenados) e acesso (quando o conteúdo é disponibilizado para os interessados) [Abowd e Mynatt 2000]. Além delas, uma quinta fase denominada "extensão" também pode ser destacada das demais [Pimentel et al. 2001]. Tal fase compreende o momento em que os usuários interagem e complementam os conteúdos capturados. De modo a sintetizar o processo de C\&A, a Figura 1 apresenta as cinco fases supracitadas.

\section{Abordagem Proposta}

Os OAs criados nas fases pré e pós-produção podem, em situações futuras, ser acessados e enriquecidos na fase de extensão por meio de atividades sociais e colaborativas, como comentários, classificação de slides e criação de bookmarks. Os OAs subdividem-se em três níveis hierárquicos: aula, tópico e slide. Uma aula consiste em vários tópicos que 


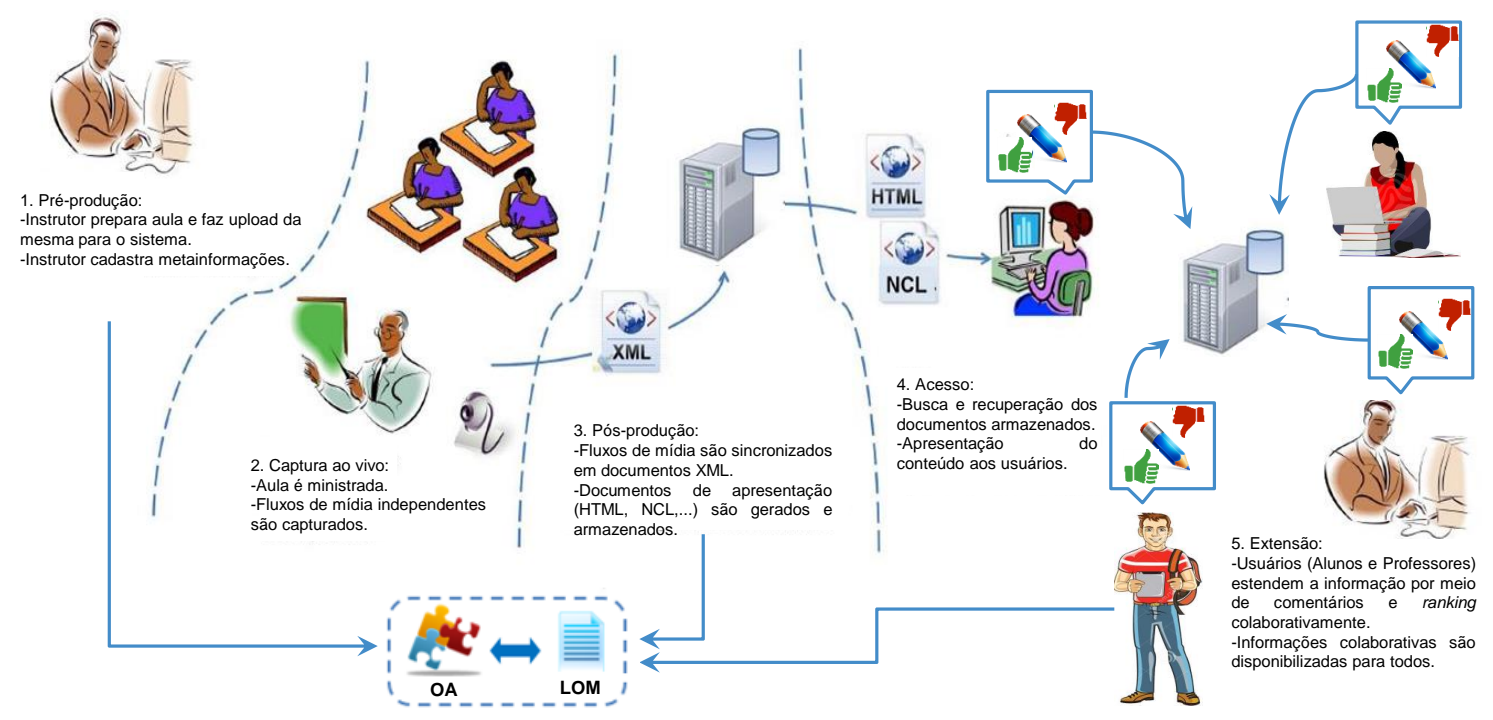

Figura 1. As 5 fases do processo de C\&A, adaptado de [Brant-Ribeiro et al. 2014].

possuem um ou mais slides associados. O OA de um slide contém o conteúdo do respectivo slide e as anotações feitas na lousa digital. Quando o professor termina uma sessão de captura em sala de aula, são criados diversos OAs: um para cada slide existente.

Ao acessar a aula capturada, estudantes e professores podem definir seus subtópicos, denominados marcadores de tópico (ou bookmarks). A funcionalidade de criação de um novo bookmark provê um mecanismo de autocompletar contextual ao digitar um título para o marcador, baseado no conjunto de bookmarks já criados por outros usuários para a aula em questão. Em complemento, também é possível definir valores para o campo de metadado "Learning Resource Type" (LRT) de cada slide indicando o tipo do seu conteúdo, tal como: enunciado, imagem, tabela, exercício, entre outros.

A abordagem proposta neste trabalho foi implementada em um AEU real, intitulado Classroom eXperience (CX) [Araújo et al. 2013]. O CX é uma plataforma educacional complementar baseada em conceitos de C\&A e utilizada para capturar, armazenar, acessar e estender o conteúdo multimídia em AEUs. Na Figura 2 é possível observar um exemplo de aula capturada e disponilizada aos estudantes. A região indicada pelo número 1 demonstra o bookmark definido naquele slide e a região 2 mostra uma caixa suspensa com as opções de tipos de conteúdo do slide. Os valores selecionados nessa caixa suspensa farão parte do campo de metadado LRT do slide e, por conseguinte, farão parte tanto dos metadados do OA do tópico em que pertencem, caso ele exista, quanto do OA da aula completa.

O conjunto de valores apresentados na caixa-suspensa de LRT é composto pelos possíveis valores definidos pelo LOM [IEEE 2002], exceto "questionário", "avaliação", "experimento", "slide" e "aula". Os valores "slide" e "aula" são determinados automaticamente pelo sistema e os outros três foram desconsiderados por não serem aderentes ao tipo de OA gerado pelo sistema. O sistema suporta o tipo "questionário", mas é um OA diferente daqueles capturados em sala de aula. Ao se passar o mouse sobre a miniatura do slide ou sobre a imagem do marcador (região indicada pelo número 2 na Figura 2), o título dado ao marcador é apresentado, caso exista. Se nenhum bookmark tiver sido definido 
V Congresso Brasileiro de Informática na Educação (CBIE 2016)

Anais do XXVII Simpósio Brasileiro de Informática na Educação (SBIE 2016)

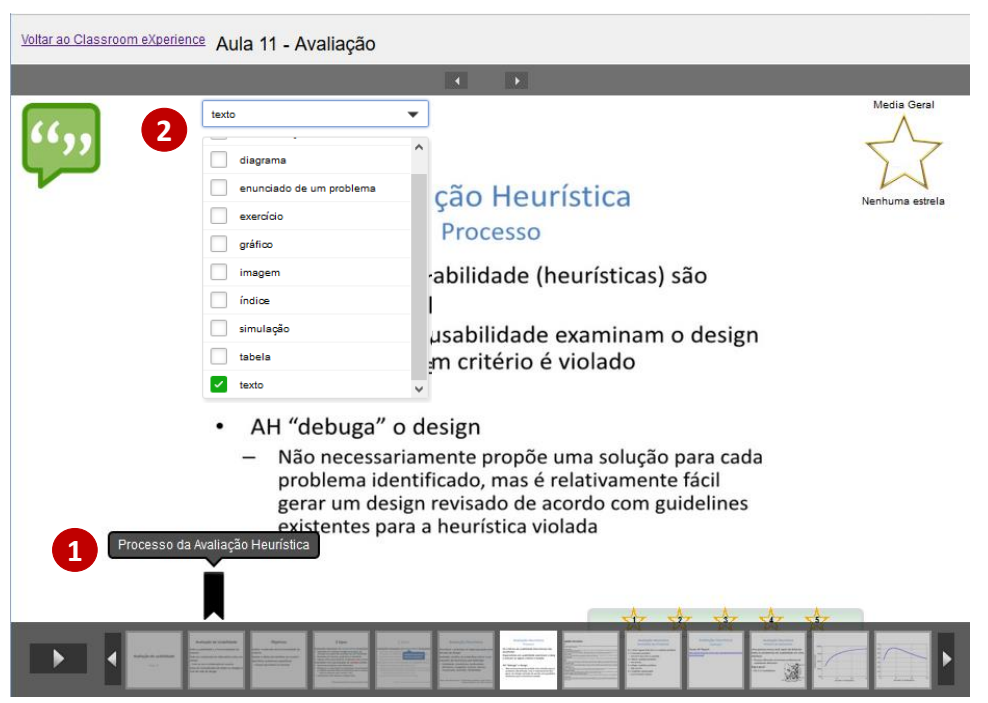

Figura 2. Definição de bookmarks numa aula capturada.

para o slide, então um ícone com o sinal de adição (“+”) é apresentado, simbolizando a criação de um novo bookmark para a aula.

Ao se clicar na imagem do bookmark, uma janela pop-up é aberta. A Figura 3(a) apresenta o caso de criação de um novo bookmark. Se um bookmark já existir e o mesmo tiver sido criado pelo usuário da sessão, um botão "excluir" será apresentado, conforme Figura 3(b). Caso o bookmark já exista, mas tenha sido criado por outro usuário, os botões "gostei" e "não gostei" serão apresentados para permitir que o usuário informe a qualidade do bookmark definido (Figura 3(c)). Além disso, o usuário poderá definir um bookmark diferente, se desejar. Assim, os bookmarks da aula serão atualizados dinamicamente de acordo com as atividades de criação, exclusão e atualização dos usuários.

A princípio, quatro regras têm sido aplicadas para a apresentação dos marcadores, na respectiva ordem: (1) bookmarks criados pelo próprio usuário; (2) opção com maior número de atividades "gostei" entre os usuários; (3) opção com maior número de bookmarks semelhantes (para efeitos de comparação, todos os títulos são convertidos para letras minúsculas); ou (4) o bookmark com data mais antiga, caso nenhuma das outras regras sejam satisfeitas. Tendo por base que a apresentação dos bookmarks acontece de maneira fracamente acoplada, a mesma poderá ser substituída por diferentes algoritmos e propostas caso surjam alternativas convenientes em circunstâncias futuras.

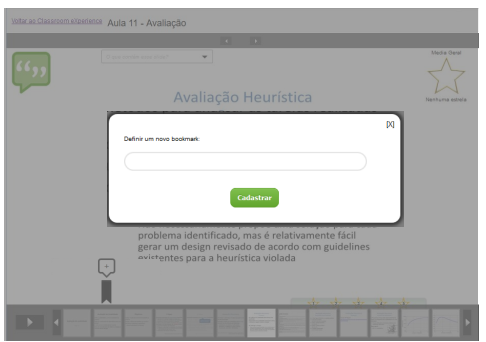

(a)

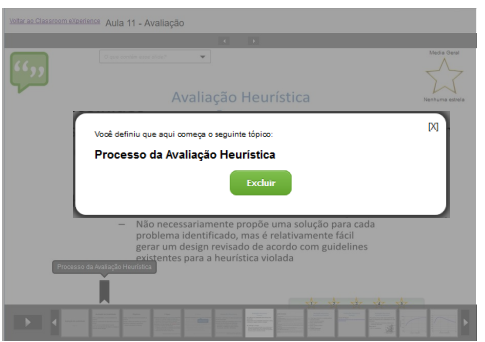

(b)

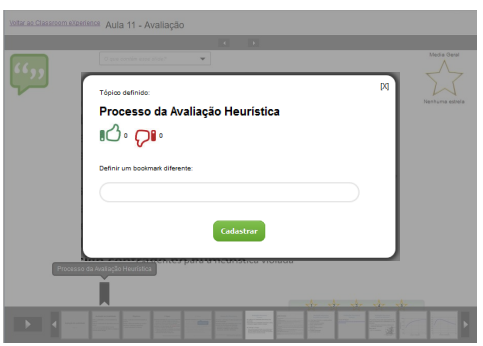

(c)

Figura 3. Criação, exclusão e avaliação de um bookmark. 
V Congresso Brasileiro de Informática na Educação (CBIE 2016)

Anais do XXVII Simpósio Brasileiro de Informática na Educação (SBIE 2016)

\section{Experimento com Usuários}

Com o objetivo de validar a proposta apresentada, foi realizado um experimento com estudantes matriculados na disciplina "Interação Humano-Computador" (IHC) oferecida aos cursos de graduação em Sistemas de Informação e pós-graduação em Ciência da Computação da Faculdade de Computação da Universidade Federal de Uberlândia. Com o intuito de corroborar os resultados obtidos, especialistas também avaliaram a qualidade dos metadados gerados durante esta etapa. No total, 30 estudantes e três especialistas da área de IHC participaram deste experimento.

\subsection{Configuração do Experimento}

O experimento realizado foi dividido em quatro fases: (I) Definição de bookmarks, (II) Avaliação de bookmarks, (III) Redefinição de bookmarks e (IV) Avaliação de especialistas. A fase I foi realizada com nove estudantes do curso de pós-graduação. As fases II e III foram realizadas com 21 estudantes de graduação e a fase IV empregou três especialistas. Duas aulas distintas da disciplina IHC foram selecionadas para a realização do experimento: "Avaliação de Usabilidade" e "Análise de Tarefas", denominadas "Aula A" e "Aula B", respectivamente. Os participantes tiveram contato prévio com o conteúdo das aulas, visto que elas foram ministradas em datas anteriores ao experimento.

Ao final das fases I, II e III, um questionário ${ }^{1}$ com oito afirmativas foi apresentado aos participantes para as avaliarem em uma Escala Likert de sete pontos. Optou-se por utilizar uma escala com sete níveis em razão da mesma possuir um intervalo maior para a atribuição de respostas, permitindo a realização de análises com o intuito de compreender a variabilidade das opiniões entre os usuários. As afirmativas foram elaboradas de acordo com conceitos de qualidade e avaliação de software [ISO/IEC 2011], conforme apresentado na Tabela 1:

Tabela 1. Critérios de avaliação das fases I, II e III.

\begin{tabular}{lll}
\hline Categoria & & Descrição \\
\hline Corretude & Percepção com relação à corretude dos bookmarks. & Fases \\
Eficácia & Precisão e integridade para os usuários atingirem os objetivos do OA. & I, II, III \\
Utilidade & Percepção de uma funcionalidade ser útil para o usuário atingir seus objetivos. \\
Navegação & Facilidade de visualização e navegação entre diferentes conceitos contidos em uma aula. & II \\
Satisfação & Nível de satisfação de um usuário em determinado contexto. & I, II, III \\
Nova funcionalidade & Possibilidade de definição de hierarquia para os bookmarks. & I, III \\
\hline
\end{tabular}

$\mathrm{Na}$ fase I, foi pedido que os participantes navegassem e definissem bookmarks para as Aulas A e B. Com o objetivo de obter dados mais precisos, os participantes foram divididos em 2 grupos, um com 5 e outro com 4 pessoas. Ao final, os marcadores criados foram agrupados e, para os marcadores sobrepostos, foram escolhidos aqueles que tiveram mais atividades de "gostei" ou escolhidos pelo professor da disciplina, em caso de empate.

$\mathrm{Na}$ fase II, a configuração seguiu as definições de experimentos intrassujeitos (within-subjects) com contrabalaceamento [Lane 2006] para minimizar os efeitos residuais. Além disso, os participantes foram divididos aleatoriamente em dois grupos para que apenas uma das duas aulas fosse vista com bookmarks por cada grupo, conforme pode

\footnotetext{
${ }^{1}$ Fases I e III: http://tinyurl.com/guhqy9x; Fase II: http://tinyurl.com/gtuevya
} 
V Congresso Brasileiro de Informática na Educação (CBIE 2016)

Anais do XXVII Simpósio Brasileiro de Informática na Educação (SBIE 2016)

ser visto na Figura 4. As aulas foram apresentadas em ordem aleatória dentro dos grupos. O objetivo desta fase foi avaliar a qualidade dos bookmarks criados na fase I e também a utilidade dos mesmos se comparados com uma aula que não possuía tais informações.

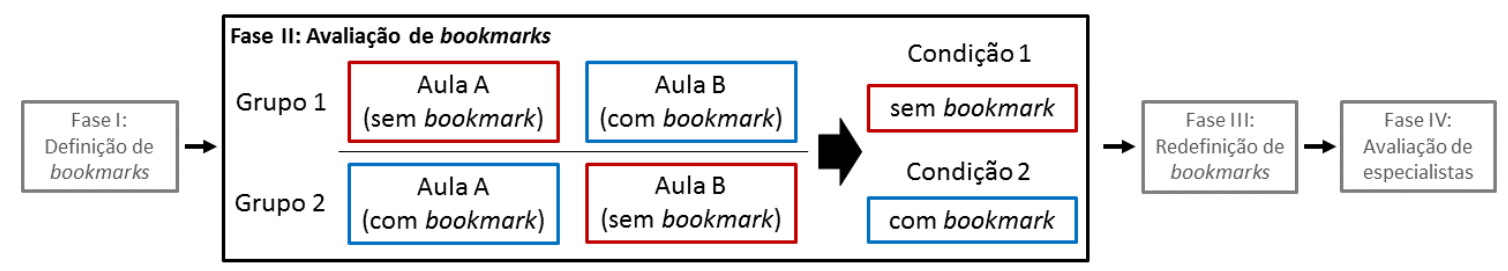

Figura 4. Configuração intrassujeitos da fase II do experimento com usuários.

Na fase III, os mesmos participantes da fase II foram convidados a acessarem a aula visualizada sem bookmarks para que pudessem definí-los. Dessa maneira, metade dos participantes definiu bookmarks para a Aula A e metade para a Aula B. Por fim, na fase IV, os OAs distintos criados nas fases I e III foram apresentados aos especialistas que avaliaram a qualidade e consistência dos metadados associados. Os OAs foram apresentados em ordem aleatória aos especialistas, sendo que os dois últimos apresentados foram os OAs das aulas completas. Para cada OA, cinco afirmativas foram apresentadas aos especialistas para que eles as respondessem em uma Escala Likert de cinco pontos, baseada no Instrumento de Avaliação de Objetos de Aprendizagem (LORI) [Nesbit et al. 2004, Vargo et al. 2003] e nos conceitos de qualidade e avaliação de software [ISO/IEC 2011], conforme Tabela 2.

Tabela 2. Questionário aplicado aos especialistas na fase IV.

\begin{tabular}{lll}
\hline & \multicolumn{1}{c}{ Afirmativas } & Categoria \\
\hline 1 & Os metadados deste OA estão consistentes com o conteúdo. & Consistência \\
2 & Todos os metadados criados estão corretos. & Corretude \\
3 & Os tópicos deste OA estão bem divididos. & Granularidade \\
4 & Este OA possui um objetivo de aprendizagem bem definido. & Alinhamento com objetivos \\
5 & É possível portar este OA para outros cursos/contextos sem modificação. & Reusabilidade \\
\hline
\end{tabular}

\subsection{Resultados e Discussão}

Durante a fase I, 54 OAs foram criados. Destes, 36 referiram-se à Aula A e 18 à Aula B. Após uma etapa de remoção de duplicatas e desambiguação realizada pelo professor da disciplina, as Aulas A e B passaram a ter, respectivamente, 24 e 12 OAs. Na fase III, foram criados 26 OAs para a Aula A e 11 OAs para a Aula B, de modo que cerca de $50 \%$ deles foram similares aos criados na fase I. Na Tabela 3 é possível observar as respostas obtidas a partir dos questionários aplicados aos estudantes envolvidos nas fases I e III.

De maneira geral, as médias das respostas obtidas em ambas as fases mantiveramse acima do ponto central da escala. Isso indica que os participantes tiveram uma tendência maior à concordância com as afirmativas expostas, evidenciando que a abordagem apresentada teve boa aceitação pelos estudantes. Em particular, é possível destacar que o recurso de auto-completar contextual (compreendido pela afirmativa 6) obteve uma das maiores taxas de aceitação entre os estudantes analisados. Isso demonstra que 
V Congresso Brasileiro de Informática na Educação (CBIE 2016)

Anais do XXVII Simpósio Brasileiro de Informática na Educação (SBIE 2016)

Tabela 3. Média, desvio padrão e coeficiente de variação das respostas dos estudantes de pós-graduação e graduação aos questionários das fases I e III.

\begin{tabular}{cccccc}
\hline Afirmativas $^{1}$ & \multicolumn{2}{c}{ Fase I } & & \multicolumn{2}{c}{ Fase III } \\
\cline { 2 - 3 } \cline { 6 - 6 } \cline { 6 - 6 } & $\bar{x} \pm s$ & $C V(\%)$ & & $\bar{x} \pm s$ & $C V(\%)$ \\
\hline 1 & $5,56 \pm 1,42$ & 25,63 & & $5,73 \pm 1,42$ & 24,80 \\
2 & $4,56 \pm 1,59$ & 34,90 & & $5,64 \pm 1,21$ & 21,40 \\
3 & $5,00 \pm 1,66$ & 33,17 & & $4,91 \pm 1,64$ & 33,42 \\
4 & $4,56 \pm 1,67$ & 36,59 & & $5,64 \pm 1,21$ & 21,40 \\
5 & $3,67 \pm 1,41$ & 38,57 & & $4,18 \pm 1,60$ & 38,29 \\
6 & $5,67 \pm 1,22$ & 21,61 & & $5,73 \pm 1,10$ & 19,27 \\
7 & $4,78 \pm 1,86$ & 38,84 & & $4,82 \pm 1,47$ & 30,53 \\
8 & $6,44 \pm 0,73$ & 11,27 & & $5,27 \pm 1,68$ & 31,84 \\
\hline
\end{tabular}

${ }^{1} \bar{x} \pm s:$ Média e desvio padrão; $C V$ : Coeficiente de variação.

tal recurso mostrou-se relevante para os participantes, pois em ambos os casos houve um índice elevado de aprovação acerca da existência desta funcionalidade.

Percebeu-se também que os estudantes da pós-graduação apresentaram um alto nível de concordância a respeito da possibilidade de se criar bookmarks em níveis hierárquicos (afirmativa 8). Neste caso, foi possível notar o maior índice de homogeneidade dentre todas respostas obtidas nesta pesquisa, resultando num $C V$ de $11,27 \% \mathrm{e}$ que evidencia a equivalência das opiniões dos participantes acerca deste tópico. Tal resultado mostra-se relevante pois, por meio desta funcionalidade, será possível indicar que determinados tópicos de um OA são subitens de outros, permitindo a geração de OAs mais concisos e estruturados. As menores avaliações em ambos os grupos referiram-se à afirmativa 5, o que indica que os estudantes podem não estar totalmente dispostos a ter de criar bookmarks para cada uma das aulas capturadas. Entretanto, os usuários não precisam obrigatoriamente utilizar a funcionalidade em todas as aulas que têm contato, visto que esta é uma proposta de caráter colaborativo e a estrutura dos OAs é criada à medida que cada usuário cria a quantidade de bookmarks que julgar conveniente.

Na Tabela 4 é possível observar as respostas de 21 estudantes de graduação que avaliaram a qualidade, utilidade e navegabilidade dos bookmarks criados. De modo geral, as médias das respostas mantiveram-se acima do ponto central da escala, indicando uma maior concordância com as afirmativas apresentadas. A maior média obtida ocorreu na

Tabela 4. Média, desvio padrão e coeficiente de variação das respostas dos estudantes de graduação ao questionário da fase II.

\begin{tabular}{ccc}
\hline Afirmativas $^{1}$ & $\bar{x} \pm s$ & $C V(\%)$ \\
\hline 1 & $5,76 \pm 1,09$ & 18,94 \\
2 & $5,62 \pm 1,47$ & 26,08 \\
3 & $5,05 \pm 1,50$ & 29,70 \\
4 & $5,95 \pm 1,07$ & 18,00 \\
5 & $4,86 \pm 1,71$ & 35,23 \\
6 & $5,43 \pm 1,40$ & 25,77
\end{tabular}

${ }^{1} \bar{x} \pm s:$ Média e desvio padrão; $C V$ : Coeficiente de variação. 
V Congresso Brasileiro de Informática na Educação (CBIE 2016)

Anais do XXVII Simpósio Brasileiro de Informática na Educação (SBIE 2016)

afirmativa 4, que compreendeu a percepção de corretude dos bookmarks definidos pelos participantes da fase I. Já a menor média obtida ocorreu na afirmativa 5, que estava relacionada à percepção de utilidade da funcionalidade para que fosse possível compreender melhor a estrutura das aulas a partir de seu emprego. De todo modo, todas as médias das respostas obtidas a partir dos questionários demonstraram resultados elevados, o que indica que os estudantes tiveram uma tendência maior a concordarem com as afirmativas apresentadas na pesquisa.

Por fim, a partir da análise das impressões dos especialistas consultados na fase IV, os valores definidos para os metadados dos OAs criados mostraram-se consistentes com o conteúdo dos mesmos. Além disso, os especialistas também pontuaram positivamente a corretude dos metadados preenchidos e o objetivo de aprendizagem de cada um deles, concordando que os bookmarks definidos foram realmente capazes de representar os tópicos abordados em ambas as aulas.

\section{Trabalhos Relacionados}

Vários trabalhos relacionados à autoria de objetos de aprendizagem e captura multimídia podem ser encontrados na literatura. Muitos deles apresentam abordagens manuais para a criação tanto dos conteúdos de OAs em si quanto de seus metadados. No survey apresentado por [Battistella e von Wangenheim 2011], algumas ferramentas de autoria de OAs foram analisadas. No entanto, nenhuma delas apresenta funcionalidades colaborativas para criação e particionamento desses objetos. Além disso, os OAs não são criados e modificados em AEUs.

LO Creator [Koohang et al. 2011], por exemplo, é uma ferramenta Open Source que permite a criação de OAs em três níveis: conceito, teoria e visão. Apesar de trabalhar com granularidades diferentes, os metadados são informados manualmente e sem nenhuma atividade colaborativa. Já o trabalho apresentado em [Osamnia et al. 2014] propõe um sistema automatizado de autoria que gera conteúdo multimídia enriquecido baseado em apresentações em tempo real. No entanto, poucos metadados são preenchidos e também não existem interações colaborativas na proposta, além de não haver algum mecanismo para segmentar o OA.

O trabalho apresentado em [Miranda e Ritrovato 2014] não se trata exatamente de uma ferramenta de autoria de OAs, mas propõe uma abordagem para extrair automaticamente seus metadados diretamente do conteúdo. Tal abordagem é baseada em heurísticas que são utilizadas para o preenchimento de 11 campos de metadados e pode ser executada em OAs de diferentes granularidades. De todo modo, a proposta do presente trabalho vai além ao permitir que os estudantes enriqueçam o conteúdo por meio de atividades colaborativas e os metadados sejam atualizados dinamicamente.

A maioria dos trabalhos encontrados na literatura foca na autoria de OAs, mas não permite o enriquecimento do conteúdo por meio de atividades colaborativas. Além disso, tais abordagens permitem a criação de OAs em diferentes granularidades, porém não provêm mecanismos para a segmentação dos OAs em granularidades mais finas.

\section{Conclusão e Trabalhos Futuros}

Objetos de Aprendizagem (OAs) têm sido cada vez mais utilizados em ambientes educacionais como suporte ao processo de ensino/aprendizagem. No entanto, a criação de tais 
V Congresso Brasileiro de Informática na Educação (CBIE 2016)

Anais do XXVII Simpósio Brasileiro de Informática na Educação (SBIE 2016)

objetos é uma tarefa árdua e, para que os OAs possam ser recuperados e reutilizados no futuro, é necessário informar metadados consistentes e em formatos padronizados. Neste contexto, Ambientes Educacionais Ubíquos (AEUs) podem ser utilizados para auxiliar a geração de OAs e melhorar a experiência de aprendizagem por meio de atividades colaborativas. Os AEUs também podem contribuir na segmentação destes OAs, dando origem a novos OAs de granularidade mais fina de modo a facilitar sua reutilização.

Este trabalho propõe uma abordagem para a segmentação de OAs em AEUs utilizando a funcionalidade de bookmarks colaborativos, que pode ser empregada tanto por estudantes quanto professores no momento em que a aula capturada é disponibilizada para o estudo. A proposta foi avaliada por meio de experimentos com usuários e especialistas e também foi implementada como estudo de caso em um AEU real. De maneira geral, as médias das respostas obtidas na avaliação mantiveram-se acima do ponto central da escala. Isso indica que os participantes tiveram uma tendência maior à concordância com as afirmativas expostas, o que evidencia a boa aceitação da abordagem pelos estudantes. Além disso, segundo os especialistas, os metadados dos OAs criados estavam corretos e consistentes com o contéudo dos mesmos.

Como trabalhos futuros, os OAs de granularidade mais fina gerados serão utilizados em algoritmos de recomendação e personalização. Abordagens capazes de considerar os Estilos de Aprendizagem dos estudantes serão investigadas para esta finalidade. Além disso, pretende-se criar um mecanismo hierárquico de OAs e integrar um modelo de gamificação para incentivar a colaboração. Por fim, os OAs criados serão disponibilizados para professores de outras disciplinas de modo a poderem ser reutilizados.

\section{Agradecimentos}

Os autores agradecem às agências brasileiras de pesquisa CNPq, CAPES e FAPEMIG, ao $\mathrm{PET} / \mathrm{SESu} / \mathrm{MEC}$ e à $\mathrm{PROPP} / \mathrm{UFU}$ pelo apoio concedido a este trabalho e às sugestões dos integrantes do laboratório PAWS da University of Pittsburgh, EUA.

\section{Referências}

Abowd, G. D. e Mynatt, E. D. (2000). Charting Past, Present, and Future Research in Ubiquitous Computing. ACM Trans. Comput.-Hum. Interact., 7(1):29-58.

Araújo, R. D., Brant-Ribeiro, T., Cattelan, R. G., de Amo, S. A., e Ferreira, H. N. M. (2013). Personalization of Interactive Digital Media in Ubiquitous Educational Environments. In Proc. of the IEEE Intern. Conf. on Systems, Man and Cybernetics, SMC'13, páginas 3955-3960.

Araújo, R. D., Brant-Ribeiro, T., de Freitas, R. S., Dorça, F. A., e Cattelan, R. G. (2014). Autoria automática de objetos de aprendizagem a partir de captura multimídia e associação a estilos de aprendizagem. In Anais do XXV Simpósio Brasileiro de Informática na Educação, SBIE'14, páginas 229-238.

Battistella, P. E. e von Wangenheim, A. (2011). Avaliação de Ferramentas de Autoria Gratuitas para produção de Objetos de Aprendizagem no padrão SCORM. Revista Brasileira de Informática na Educação, 19(2):16-28.

Braga, J. C., Dotta, S., Pimentel, E., e Stransky, B. (2012). Desafios para o Desenvolvimento de Objetos de Aprendizagem Reutilizáveis e de Qualidade. In Anais do Workshop de Desafios da Computação Aplicada à Educação, DesafIE, páginas 90-99. 
V Congresso Brasileiro de Informática na Educação (CBIE 2016)

Anais do XXVII Simpósio Brasileiro de Informática na Educação (SBIE 2016)

Brant-Ribeiro, T., Mendonça, I. E. S., Araújo, R. D., Mendes, M. M., Dorça, F. A., e Cattelan, R. G. (2014). Um Modelo Social e Colaborativo para Extensão de Conteúdo em Ambientes Educacionais Ubíquos. Tecnologias, Sociedade e Conhecimento, 2(1):105130, NIED.

Dias, C. L., Kemczinski, A. L., Sá S. V.; Ferlin, J., e Hounsell, M. S. (2009). Padrões abertos: aplicabilidade em Objetos de Aprendizagem (OAs). In Anais do XX Simpósio Brasileiro de Informática na Educação, SBIE'09.

IEEE (2002). Draft Standard for Learning Object Metadata. Learning Technology Standards Committee of the IEEE. IEEE LTSC 1484.12.1.

ISO/IEC (2011). ISO/IEC 25010:2011 - Systems and software engineering - Systems and software Quality Requirements and Evaluation (SQuaRE) - System and software quality models. Technical report.

Koohang, A., Floyd, K., e Stewart, C. (2011). Design of an Open Source Learning Objects Authoring Tool - The LO Creator. Interdisciplinary Journal of E-Learning and Learning Objects, 7:111-123.

Lane, D. M. (2006). Online Statistics Education: An Interactive Multimedia Course of Study. Disp. em http://onlinestatbook.com. Proj. Leader: David M. Lane, Rice Univ.

Miranda, S. e Ritrovato, P. (2014). Automatic Extraction of Metadata from Learning Objects. In Proc. of the Intern. Conf. on Intelligent Networking and Collaborative Systems, INCoS, páginas 704-709.

Nesbit, J., Belfer, K., e Leacock, T. (2004). LORI 1.5: Learning Object Review Instrument. Technical report. Acessado em 15 de maio de 2016. Disponível em http://www.transplantedgoose.net/gradstudies/educ892/LORI1.5.pdf.

Osamnia, M., Chunwijitra, S., Berena, A. J., Okada, H., e Ueno, H. (2014). A Cloudbased Automated Authoring System to Support e-Learning in Higher Education Under Low-Speed Internet. Int. Journal of Advances in Computer Science \& Its Applic., 4(3).

Pimentel, M. d. G., Ishiguro, Y., Kerimbaev, B., Abowd, G. D., e Guzdial, M. (2001). Supporting Educational Activities through Dynamic Web Interfaces. In Interacting with Computers, Special Issue on Interacting with the Active Web, páginas 353-374.

Solis, A. M. e Laviste, R. P. (2015). Development and Evaluation of Electronic Learning Objects for Digital Electronics. International Journal of Mathematics, Engineering \& Technology, 11(1):124-144.

Vargo, J., Nesbit, J., Belfer, K., e Archambault, A. (2003). Learning Object Evaluation: Computer-Mediated Collaboration And Inter-Rater Reliability. Intern. Journal of Computers and Applications, 25(3):198-205.

Wiley, D. A. (2002). Connecting learning objects to instructional design theory: A definition, a metaphor, and a taxonomy, páginas 3-23. The Agency for Instructional Technology, Bloomington, Indiana, USA. Acessado em 10/05/2016, http://reusability.org/read/chapters/wiley.doc.

Zhao, X., Wan, X., e Okamoto, T. (2010). Adaptive Content Delivery in Ubiquitous Learning Environment. In Proc. of the 6th IEEE Intern. Conf. on Wireless, Mobile and Ubiquitous Technologies in Education, WMUTE'10, páginas 19-26. 